\title{
Epidemiology and rheumatology: how can epidemiologists affect treatment strategies?
}

\author{
Deborah PM Symmons
}

It is a widely held view that an epidemiologist's main task is to study the incidence and prevalence of disease. Often invited to speak in the first sessions at symposia, epidemiologists 'set the scene' by describing how many people have the disease in question, and their distribution by place and time. When it comes to the sessions on treatment, these experts seldom take to the podium; however, epidemiologists have a tremendous amount to offer in the design of treatment strategies.

Most basic research scientists and those in charge of clinical trials address single issues. The basic scientist focuses, for example, on the function of a particular cytokine or gene in the etiology or pathogenesis of a disease. Clearly, however, most rheumatic diseases involve complex mechanisms; they are caused by the interaction of a number of genes and environmental factors. Often, many different etiological pathways lead to the same disease phenotype; it is the genetic epidemiologist who takes the lead in discerning these pathways. Similarly, randomized controlled trials evaluate a single drug or drug combination. Unfortunately, the results of a randomized controlled trial only inform the investigating rheumatologist about the response of the 'average' patient to that particular drug or drug combination, and not how to treat the individual patient in his or her clinic. As an example, there are now many trials demonstrating that, on average, antitumor necrosis factor (TNF) therapy is better than methotrexate for the treatment of severe rheumatoid arthritis, psoriatic arthritis and ankylosing spondylitis. There are no trials, however, that compare one anti-TNF agent with another, or that establish which patients will respond to anti-TNF therapy and which will not. These are issues that, whether for commercial, economic or practical reasons, cannot be addressed in
". . "however,

have a

tremendous

amount to offer

in the design

of treatment

strategies

DPM Symmons

is a Professor of

Rheumatology and

Musculoskeletal

Epidemiology

in the Arthritis

Research Campaign

Epidemiology Unit

at the University

of Manchester,

Manchester, UK.

\section{Competing interests}

The author declared no

competing interests.

www.nature.com/clinicalpractice doi:10.1038/ncprheum0703 the trial setting. Instead, there is a need for large observational studies, which carefully collect information on treatment choice (and the reasons for it), disease characteristics, genetic and other biomarkers, demographic and psychosocial factors, compliance and response. Neither the collection nor the analysis of such datasets is a trivial matter.

The collection of data requires the commitment of large numbers of rheumatologists. The information must be collected from as many and as diverse clinical settings as possiblefrom the tiny, single-handed practice to the huge teaching hospital-because different patient groups and responses to treatment might be seen in each of these environments. Rheumatologists need to be trained (by epidemiologists) to collect data in a complete and standardized fashion; this means that they must think carefully about the way that they apply diagnostic labels, and treat patients according to prespecified protocols or record why they have deviated from their own standard practice. The epidemiologist (who needs a background in clinical rheumatology) can then apply complex statistical modeling, which should include terms for the interaction between the variables under study, to identify predictors of treatment response.

This approach is now needed in most fields of rheumatology. Systematic reviews and meta-analyses (which are also the domain of the epidemiologist) might help to some extent, but there are few diseases in which these approaches lead to a single treatment choice that will be effective for every patient. Internationally, there might not be enough rheumatology-trained epidemiologists to go round-and this needs to be remedied. We are aiming not so much for 'bench to bedside', but rather 'computer to couch'. 